\title{
How to win the ovarian cancer stem cell battle: destroying the roots
}

\author{
Akimasa Takahashi ${ }^{1,2}$, Linda Hong ${ }^{3}$, and Ilana Chefetz ${ }^{1,4,5,6}$ \\ 'The Hormel Institute, University of Minnesota, Austin, MN 55912, USA. \\ 2Department of Obstetrics and Gynecology, Shiga University of Medical Science, Otsu, Shiga 5202152, Japan. \\ ${ }^{3}$ Department of Obstetrics \& Gynecology, Division of Gynecologic Oncology, Loma Linda University School of Medicine, CA \\ 92350, USA. \\ ${ }^{4}$ Masonic Cancer Center, Minneapolis, MN 55455, USA \\ ${ }^{5}$ Stem Cell Institute, Minneapolis, MN 55455, USA \\ ${ }^{6}$ Department of Obstetrics, Gynecology and Women's Health, Minneapolis, MN 55455, USA.
}

Correspondence to: Asst. Prof. Ilana Chefetz, The Hormel Institute, University of Minnesota, 80116th Ave NE Austin MN 55912 USA.E-mail: ichefetz@umn.edu

How to cite this article: Takahashi A, Hong L, Chefetz I. How to win the ovarian cancer stem cell battle: destroying the roots. Cancer Drug Resist 2020;3:1021-33. http://dx.doi.org/10.20517/cdr.2020.93

Received: 9 Oct 2020 First Decision: 20 Nov 2020 Revised: 30 Nov 2020 Accepted: 3 Dec 2020 Available online: 22 Dec 2020

Academic Editors: Godefridus J. Peters, Paola Perego Copy Editor: Monica Wang Production Editor: Jing Yu

\begin{abstract}
Ovarian cancer has the highest mortality rate among gynecologic malignancies. The combination of cytoreductive surgery and chemotherapy is the standard regimen for the treatment of ovarian cancer. The initial treatment is usually effective, but many patients with ovarian cancer experience recurrence, and treatment options for recurrent disease remain challenging. Cancer stem cells (CSCs) are suggested to play an essential role in cancer recurrence after initial chemotherapy. Furthermore, they are of great interest as CSCs may also be involved in chemotherapy susceptibility. Thus, understanding the characteristics and mechanisms by which CSCs display resistance to therapeutic agents is important to design effective cancer treatments. In this review, we describe and discuss current therapeutic regimens for ovarian cancer, as well as the various CSC markers, association between CSCs and disease progression, correlation of CSCs with poor prognosis, enrichment of CSCs in tumor tissues following repeated chemotherapy cycles, activation of major signaling pathways following chemotherapy, and potential inhibitors that suppress these signaling cascades. In addition, clinical trials evaluating novel targeted therapies to overcome chemotherapy resistance will be reviewed. The combination of traditional chemotherapy and CSCtargeted therapy could be an effective and promising anticancer treatment for ovarian cancer. Understanding the biological properties of CSCs and the mechanism of chemotherapy resistance are critical to design and develop new therapeutic strategies to overcome CSC-associated chemotherapy resistance.
\end{abstract}

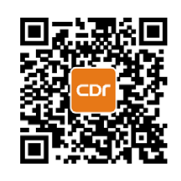


Keywords: Ovarian cancer, platinum resistance, cancer stem cell, chemotherapy, targeted therapy

\section{INTRODUCTION}

Ovarian cancer is the leading cause of gynecologic cancer-related death. Optimal cytoreductive surgery in combination with chemotherapy is the standard treatment for ovarian cancer. After first-line chemotherapy with carboplatin and paclitaxel, $80 \%$ of ovarian cancer patients will initially respond. However, $80 \%$ of advanced-stage patients and $20 \%$ of early-stage patients will eventually relapse. Recent tumorigenesis hypothesis suggests that cancer stem cells (CSCs) remaining in the tumor after chemotherapy are able to initiate and propagate tumors causing recurrence and chemotherapy resistance. In this review, we describe the current knowledge of ovarian cancer therapeutic options, the association between CSC expression and prognosis, chemotherapy-associated CSC enrichment, and the signaling pathways involved. Finally, we discuss the targeted cancer therapy approaches currently in clinical trials based on their effect on CSCs.

\section{OVARIAN CANCER AND THERAPEUTIC OPTIONS}

Epithelial ovarian cancer (EOC) has the highest morbidity and mortality rate among the gynecologic malignancies in developed countries ${ }^{[1]}$. In the United States, an estimated 22,240 women were diagnosed with ovarian cancer in 2018, and the age-adjusted mortality rate in 2018 was 6.27 deaths per 100,000 people $^{[2]}$. Three quarters of EOC cases are classified into four major histologic subtypes, including serous, endometrioid, clear cell, and mucinous ovarian cancer, with the remaining $25 \%$ representing rare or unspecified subtypes ${ }^{[3]}$. Ovarian cancer is categorized as type I and II and thought to be mediated by different signaling pathways. Type I cancers comprise low-grade serous, low-grade endometrioid, clear cell, mucinous cancers, and Brenner tumors and contain mutations in common oncogenes such as kirsten rat sarcoma 2 viral oncogene homolog (KRAS), B-raf proto-oncogene (BRAF), erb-b2 receptor tyrosine kinase 2 (ERBB2), catenin beta 1 (CTNNB1), phosphatase and tensin homolog (PTEN), phosphatidylinositol 3-kinase catalytic subunit alpha isoform (PIK3CA), and AT-rich interacting domain containing protein $1 A(A R I D 1 A)^{[4]}$. Type II cancers include aggressive malignancies, such as high grade serous carcinomas, carcinosarcoma, and undifferentiated carcinomas with mutations most notable in tumor protein 53 (TP53) and breast cancer susceptibility gene $(B R C A)^{[4]}$. Population-based studies have indicated that approximately $14 \%$ of ovarian cancer patients have the germline BRCA1 and BRCA2 mutations ${ }^{[5]}$. Over $75 \%$ of the ovarian cancer patients present with advanced stage disease, as defined by the spread of the disease outside the pelvis ${ }^{[6,7]}$. As a result, the 5-year survival rate of all ovarian cancer patients is $47 \%^{[2]}$. A combination of debulking surgery and chemotherapy is essential for the treatment of ovarian cancer. While the main purpose of primary surgery is to completely remove all macroscopically visible disease ${ }^{[8]}$, chemotherapy is necessary as the majority of patients will relapse despite nearly complete resection ${ }^{[9]}$. Platinum-based antineoplastic agents have been key therapeutic options during the past three decades ${ }^{[10]}$. Following first-line chemotherapy with carboplatin and paclitaxel, about $80 \%$ of ovarian cancer patients will respond and $40 \%-60 \%$ of patients will achieve complete remission ${ }^{[11]}$. Unfortunately, even for stage I or II patients, recurrence occurs in $20 \%-25 \%$ cases; whereas more than $80 \%$ of patients with advanced disease will recur ${ }^{[12]}$. The majority of patients with advanced ovarian cancer will experience disease relapse within 2 years of firstline treatment ${ }^{[13]}$, emphasizing an urgent unmet medical need for novel therapies. Recently, a combination of two targeted therapies for advanced ovarian cancer has been approved that includes a humanized antivascular endothelial growth factor (VEGF) monoclonal antibody and poly [adenosine diphosphate (ADP)ribose] polymerase (PARP) inhibitor. Randomized clinical trials have shown significant benefits in terms of progression-free survival for concomitant use of these agents with standard chemotherapy or as single agents in maintenance regimens ${ }^{[14-16]}$. In particular, the presence of mutations in $B R C A 1 / 2$ genes, which play an important role in homologous recombination repair of DNA double-strand breaks, sensitize tumor cells to various PARP inhibitors (PARPi), such as olaparib, veliparib, rucaparib, niraparib, and veliparib ${ }^{[17]}$. 
Ovarian cancer recurrence is mostly incurable; however, overall survival generally depends on platinum sensitivity. In general, patients who relapse more than 6 months after initial therapy are defined as "platinum-sensitive", patients who relapse within 6 months are characterized as "platinum-resistant", and patients who fail to respond or progress during initial treatment are defined as "platinum refractory" Patients with "platinum-sensitive" disease are treated with platinum doublet chemotherapy similarly to the initial treatment, but the vast majority of these patients will recur creating a progressive resistance to treatment, resulting in depletion of available treatment options. The main objective of treatment in recurrent disease is palliative, with a focus on controlling symptoms, prolonging survival, and improving quality of life ${ }^{[19]}$. Patients with recurrent ovarian cancer are commonly treated with a second line of nonplatinum agents, such as paclitaxel, topotecan, gemcitabine, and pegylated liposomal doxorubicin to improve the survival prognosis of "platinum-refractory" and "platinum-resistant" patients ${ }^{[20]}$.

\section{OVARIAN CANCER STEM CELLS}

CSCs represent a small subset of an inherently chemoresistant population of cancer cells. In addition to the ability of self-renewal and differentiation due to ability to divide symmetrically and asymmetrically, CSCs have the ability to initiate and propagate tumors. Due to their slow cell cycle progression, CSCs are characterized by inherent resistance to standard radio- and chemotherapies ${ }^{[2,22]}$. Chemoresistance in recurrent ovarian cancer is associated with a higher frequency of CSCs in comparison to primary ovarian cancer $^{[23]}$. The presence of cells with stem-like properties in cancer was first described in studies of human acute myelogenous leukemia ${ }^{[24]}$ in which leukemia-initiating cells were isolated based on the expression of cell surface markers $\mathrm{CD} 34^{+}$and $\mathrm{CD} 38^{+}$. Subsequently, cell surface marker-based purification methods are now commonly used to isolate CSCs from heterogeneous solid tumor cells ${ }^{[25,26]}$.

The first CSCs in ovarian cancer were identified in the ascites of EOC patients. These cells, characterized by $\mathrm{CD} 44^{+} / \mathrm{CD} 117^{+}$expression, developed tumors in mice continuously for several generations ${ }^{[27]}$. Afterwards, isolation of CSCs in ovarian cancer has been explored in various ways, including side population (SP) cells with expression of adenosine triphosphate (ATP)-binding cassette (ABC) transporters, the use of cell surface markers, and detection of aldehyde dehydrogenase activity (ALDH) by using the ALDEFLUOR fluorescence assisted cell sorting (FACS) method. Expression of various cell surface markers such as CD44, CD117, CD133, CD24, and epithelial cell adhesion molecule (EpCAM) $)^{[28-37]}$ has been reported in ovarian cancer. SP cells are characterized by the exclusion of the Hoechst 33342 dye through the ABC transporters, which transport a wide range of substrates such as drugs, metabolic products, nutrients, and lipids across extracellular and intracellular membranes. SP cells exhibit high self-renewal and proliferative capacity in vitro and possess typical CSC properties associated with tumor initiation in vivo and acquired resistance to chemotherapy as a result of shuffling out chemotherapeutic drugs by the $\mathrm{ABC}$ transporters ${ }^{[38,39]}$. However, isolation of SP cells has low specificity, and the purity of isolated cells is not sufficient compared to other methods due to high heterogeneity.

CD44 is a cell surface adhesion receptor and $\mathrm{CD} 44^{+}$cells have been reported to be present in primary and metastatic ovarian tumors as well as in cells of malignant ascites ${ }^{[27,40]}$. CD $44^{+}$cells, isolated from ascites and solid tumors, are characterized by constitutive nuclear factor-kappa B (NF- $\kappa \mathrm{B})$ activity, cytokine and chemokine production, high capacity of tumor repair and self-renewal, and resistance to conventional chemotherapy, which are unique features of $\mathrm{CSC}^{[41]}$. Myeloid differentiation protein 88 (MyD88) is an adapter protein required for toll-like receptor (TLR) signal transduction as part of an inflammatory response to bacterial and viral infection. MyD $88^{+}$cells in ovarian cancer primary cells and cell lines are resistant to paclitaxel, a known TLR4 ligand ${ }^{[42]}$ and are characterized by secretion of proinflammatory cytokines. Also, a small number of $\mathrm{CD} 44^{+} / \mathrm{MyD}_{8} 8^{+} \mathrm{EOC}$ cells can initiate tumors, suggesting CSC properties. In addition to TLR4, CD $44^{+} / \mathrm{MyD} 88^{+}$cells express TLR2, which can be activated by injury (surgery) and chemotherapy, creating a pro-inflammatory microenvironment that further enhances ovarian 
Table 1. Tumorigenicity in in vivo model by ovarian cancer stem cell markers

\begin{tabular}{|c|c|c|c|c|c|}
\hline CSC marker & Combination & Mode of CSC injection & Number of cells & Latency & Ref. \\
\hline \multirow[t]{3}{*}{ CD44 } & & $\mathrm{SC}$ & $1 \times 10^{6}$ & 6-8 weeks & [40] \\
\hline & CD117(+) & SC & $1 \times 10^{2}$ & 52-93 days & [28] \\
\hline & CD24(+), EpCAM(+) & SC & $1 \times 10^{2}$ & 5 weeks & [31] \\
\hline CD117 & $\begin{array}{l}\text { Lineage } \\
\text { (CD2, CD3, CD10, } \\
\text { CD16, CD31, CD64)(-) }\end{array}$ & SC & $1 \times 10^{2}$ & $100-128$ days & [52] \\
\hline \multirow[t]{3}{*}{ CD133 } & & SC & $1 \times 10^{2}$ & 99 days & [29] \\
\hline & $\mathrm{ALDH}(+)$ & SC & $2 \times 10^{3}$ & Not shown & [32] \\
\hline & $\mathrm{ALDH}(+)$ & SC & 30 & Not shown & [47] \\
\hline CD24 & & SC & $5 \times 10^{3}$ & 73 or 89 days & [51] \\
\hline ALDH & & SC & $1 \times 10^{2}$ & Not shown & [47] \\
\hline SP cells & & IP & $5 \times 10^{4}$ & 30-60 days & [39] \\
\hline
\end{tabular}

CSC: cancer stem cell; SP: side population; ALDH: aldehyde dehydrogenase; SC: subcutaneous injection; IP: intraperitoneal injection

CSC repair and self-renewal ${ }^{[37]}$. However, $\mathrm{CD} 44^{+}$is also expressed by many non-cancer cells in tumors, such as immune cells and vascular endothelial cells, suggesting a need to combine CD44 with additional markers to differentiate between different populations residing in tumors ${ }^{[11,43]}$. Thus, despite multiple studies showing that $\mathrm{CD}_{4} 4^{+}$cells have $\mathrm{CSC}$ properties, $\mathrm{ALDH}^{+} / \mathrm{CD} 133^{+}$double positive cells have been reported to be more abundant in recurrent tumors ${ }^{[32]}$. $\mathrm{CD}_{133^{+}}$, which was initially identified as a CSC marker in human glioblastoma ${ }^{[26]}$, is one of the best characterized cell surface markers in ovarian cancer ${ }^{[29,44]}$. Whereas $\mathrm{CD}_{133^{+}}$ovarian CSCs have exhibited tumor initiation, self-renewal, and chemoresistance capacity, not all ovarian cancer cell lines express CD133, and a few recent studies reveal no established association between

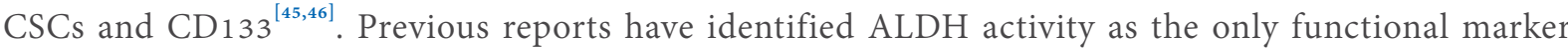
present in all ovarian cancer cell lines ${ }^{[47]}$. ALDH ${ }^{\text {high }}$ (cells with high ALDH activity) cells detected by the ALDEFLUOR FACS assay exhibit CSC capacities, as high ALDH activity correlates with higher sphereformation ability, tumorigenicity, and invasiveness ${ }^{[48,49]}$. In addition, $\mathrm{ALDH}^{\text {high }} / \mathrm{CD} 133^{+}$cells are capable of forming larger tumors during shorter time periods in mouse xenografts and also efficiently form threedimensional spheres ${ }^{[50]}$ compared to other cell populations. Existence of $\mathrm{ALDH}^{\text {high }} / \mathrm{CD}_{13} 3^{+}$in debulked primary ovarian tumor specimens is associated with worse prognosis than the existence of ALDH ${ }^{\text {low }}$ and $\mathrm{ALDH}^{\text {high }} / \mathrm{CD}_{133^{-}}$populations ${ }^{[47]}$. Comparing tumor initiation capacity, we found that at least $100 \mathrm{CD} 44$, CD24, CD133, ALDH, or SP single positive cells are required to initiate tumors in mouse xenograft models ${ }^{[29,39,40,47,51]}$; whereas fewer of $\mathrm{CD} 44^{+} / \mathrm{CD} 117^{+}$cells, $\mathrm{CD} 44^{+} / \mathrm{CD} 24^{+} / \mathrm{EpCAM}^{+}$cells, or $\mathrm{CD} 117^{+} /$lineage cells ${ }^{[2,31,52]}$ were required to form tumors when combination of two or three markers was used to isolate cells. Importantly, only $30 \mathrm{ALDH}^{+} / \mathrm{CD}_{133^{+}}$cells in murine models of ovarian cancer are sufficient to form tumors subcutaneously ${ }^{[47]}$ [Table 1]. Therefore, ALDH and CD133 are defined as a set of functionally important markers that identify ovarian CSCs.

Nanog homeobox (NANOG), octamer-binding transcription factor 4 (OCT4), and sex-determining region Y-box 2 (SOX2) are transcription factors and stemness markers that maintain pluripotency and self-renewal of embryonic and induced pluripotent stem cells. These factors are known to be commonly expressed in ovarian $\operatorname{CSCs}^{[37,53,54]}$ While many CSC marker candidates have been established, we hypothesize that different sub-populations exhibit various functional roles, which suggests a need for more systematic characterization.

\section{ASSOCIATION OF OVARIAN CSCs WITH POOR PROGNOSIS}

Various studies have identified the association between CSCs and a shorter progression-free interval and worse prognosis in ovarian cancer ${ }^{[55,56]}$, including a recent meta-analysis study that examined the correlation between four representative ovarian CSC marker candidates ALDH1/CD44/CD117/CD133 and prognosis ${ }^{[57]}$. In this meta-analysis, the increased ALDH1, CD44, and CD117 levels as assessed 
by immunochemistry were associated with poor prognosis, whereas CD133 was not. In addition, the overexpressed ALDH1 and CD44 were correlated with worse progression, but CD117 and CD133 were not ${ }^{[57]}$. Thus, CSC markers can be useful as predictive or prognostic biomarkers of ovarian cancer. In addition, high expression of EpCAM in ovarian cancer was associated with tumor recurrence and poor prognosis ${ }^{[58]}$. The disadvantage of this study was that only single CSC markers were examined. For instance, ALDH activity in combination with CD133 has revealed strong association with poor patient prognosis ${ }^{[32,47]}$. Furthermore, $\mathrm{CD} 44^{+} / \mathrm{CD} 24^{-}$expression correlates with increased recurrence rate and reduced progression-free survival in ovarian cancer patients ${ }^{[33]}$. Stemness and pluripotency factors such as OCT4, nanog, and cellular myelocytomatosis oncogene (c-Myc) have been shown to be expressed in ascites and tumor samples of ovarian cancer patients ${ }^{[59]}$. The level of OCT4 and RNA-binding protein Lin2s have been correlated with tumor malignancy and increased tumor growth ${ }^{[60]}$. Additional studies revealed the role of SOX2 in tumorigenicity, migration, and invasion as well as chemoresistance ${ }^{[53]}$. High expression of SOX2 has been associated with a significantly reduced overall survival in ovarian cancer patients, but no association between nanog or OCT4 expression and overall survival was identified ${ }^{[54]}$. Similar to OCT4 and $\mathrm{SOX} 2, \mathrm{c}-\mathrm{Myc}$ has characteristics of stemness and pluripotency, and is overexpressed not only in ovarian cancer but also in most types of malignant tumors ${ }^{[61,62]}$. In the integrated analysis of ovarian cancer by the cancer genome atlas (TCGA), c-Myc amplification was identified in $30 \%-60 \%$ of ovarian tumors ${ }^{[63]}$. This analysis revealed that disease-free survival and overall survival were decreased in ovarian cancer patients with high levels of $c-M y c$ mRNA $^{[64]}$.

\section{REPETITIVE CYCLES OF CHEMOTHERAPY AND CANCER STEM CELL ENRICHMENT}

In ovarian cancer, chemotherapy decreases tumor burden but often results in enrichment of ovarian CSCs in residual tumors ${ }^{[6]}$. Increased mRNA levels of CD44, EpCAM, and Oct4 have been observed in ascitesderived cells following multiple doses of cytotoxic chemotherapeutic agents ${ }^{[66]}$. In studies that compared CSC markers in matched primary and recurrent tumors, the expression of ALDH1A1, CD44, and CD133 markers was higher in recurrent tumors compared to primary tumors ${ }^{[67]}$. In an experimental study, ovarian cancer cells treated with the combination of paclitaxel and carboplatin exhibited enrichment of CD117 ${ }^{+}$ and $\mathrm{CD}_{133^{+}} \mathrm{CSCs}^{[68]}$. Furthermore, short-term administration of cisplatin and paclitaxel resulted in the enrichment of the OCT4 and $\mathrm{CD}_{117^{+}}$ovarian CSCs in vivo as assessed by the quantitative polymerase chain reaction $(\mathrm{qPCR})^{[69]}$. In another experiment, cell cycle-arrested cells demonstrated overexpression of OCT4, nestin, CD117, and CD44 markers following cisplatin treatment ${ }^{[70]}$.

Recently, the Food and Drug Administration (FDA) approved several PARPi for the treatment of recurrent ovarian cancer in patients with BRCA-proficient or -deficient tumors. While analysis of CSCs in clinical samples following PARPi has to be determined, in vitro and in vivo studies exhibited enriched $\mathrm{CD} 133^{+}$and $\mathrm{CD}_{117^{+}}$ovarian CSCs following PARP inhibition ${ }^{[7]}$. These data suggest that CSC enrichment can occur both after conventional antineoplastic chemotherapy as well as novel targeted therapies, suggesting a need for novel combination regimens that include CSC-targeted therapies.

\section{REPETITIVE CYCLES OF CHEMOTHERAPY AND ACTIVATION OF SIGNALING PATHWAYS}

Several key signaling pathways have been associated with stemness and self-renewal, including winglessrelated integration site (Wnt)/ $\beta$-catenin, Hedgehog, Notch, phosphatidylinositol 3-kinase (PI3-K)/ phosphatase and tensin homolog deleted chromosome 10 (PTEN), and NF- $\mathrm{BB}$ pathways. Upregulation of c-kit, a stem cell-associated receptor tyrosine kinase, causes upregulated mRNA expression of genes from the Wnt/ $\beta$-catenin pathway, subsequently leading to increased expression of $\mathrm{ABC}$ subfamily $\mathrm{G}$ member 2 (ABCG2) in ovarian CSCs, suggesting drug efflux from tumors ${ }^{[72]}$. Also, intraperitoneal administration of taxane in an in vivo mouse model triggered activation of Wnt signaling in tumor specimens and enriched expression of CSCs. Wnt antagonists, vantictumab and ipafricept, strongly block paclitaxel-mediated 
mitosis and promote mitotic cell death, effectively alleviating CSC expression. As a result, a combination regimen comprised of paclitaxel and Wnt inhibitors effectively reduced CSC content and tumor growth ${ }^{[73]}$. Activation of smoothened (SMO), patched (PICH), and hedgehog glioma-associated oncogene 1 (GLI1) in the Hedgehog signaling pathway has been reported in EOC cells. In particular, SMO and GLI1 proteins are expressed in cisplatin-resistance cancer cell lines, suggesting their role in chemoresistance ${ }^{[7]}$. Notch3, which activates the NOTCH signaling pathway, is overexpressed in over $20 \%$ of high-grade serous ovarian cancer tumors, and its upregulation is correlated with tumor recurrence, chemoresistance, and poor prognostic outcome ${ }^{[75,76]}$. Notch3 activation has been suggested to be involved in chemoresistance by upregulating expression of stem cell markers such as nanog, OCT4, kruppel-like factor 4 (KLF4), reduced expression-1 (REX1), replication timing regulatory factor 1 (RIF1), sal-like protein 4 (SALL4), and nucleus accumbens-associated 1 (NAC1 $)^{[75]}$. Interestingly, over $30 \%$ of high-grade serous ovarian tumors are associated with PTEN loss ${ }^{[77]}$, which subsequently triggers the activation of the PI3-K/protein kinase B (AKT) pathway, leading to uncontrolled cell cycle progression, diminished apoptosis, and increased metastatic disease in ovarian cancer ${ }^{[78]}$. D-116883, a PI3-K inhibitor, inhibits phosphorylation of AKT that results in Go cell cycle arrest, eventually causing apoptosis in the A2780 platinum-resistant cell line ${ }^{[79]}$. In addition, inhibitors of PI3-K/AKT signaling in combination with carboplatin have been shown to trigger apoptotic cell death in vitro and reduced ovarian cancer cell tumorigenesis in vitro and in vivo ${ }^{[80]}$. Levels of a PI3-K downstream target, phospho p70-ribosomal protein S6 kinase beta-1 (S6K), have been shown to be significantly higher in ascites of ovarian cancer patients who did not respond to subsequent chemotherapy ${ }^{[74]}$. Therefore, activation of the PI3-K pathway is potentially involved in acquired platinum resistance in ovarian cancer ${ }^{[8,82]}$. As we mentioned earlier, ovarian CSCs are characterized by an activated NF- $\kappa B$ pathway and enhanced secretion of inflammatory cytokines ${ }^{[40,83]}$. Aurora A kinase (Aurora A), a key cell cycle protein, which has a functional role in mitosis and meiosis, is overexpressed in ovarian cancer ${ }^{[84]}$. Aurora A inhibitors reduce cell proliferation in EOC CSCs by inducing cell cycle arrest and attenuating $\mathrm{NF}-\kappa \mathrm{B}$ activity ${ }^{[85]}$. Thus, inhibiting various signaling pathways directly or indirectly can ameliorate tumorigenesis and prevent cancer recurrence.

\section{COMBINATION REGIMENS: CHEMOTHERAPY AND CSC-TARGETED THERAPIES}

Two potential alternative therapeutic approaches could be used in ovarian cancer to eliminate CSCs. In the first approach, CSCs would be differentiated into non-CSCs followed by treatment with chemotherapy; while in the second approach CSCs are directly targeted by inhibiting the CSC-associated signaling pathways. For example, all-trans retinoic acid (ATRA), a vitamin A derivative that is used as a stem cell differentiation therapy in acute promyelocytic leukemia, contributes to the high remission rate in these patients ${ }^{[86]}$. ATRA is known to play an important role in cellular proliferation, differentiation, and apoptosis $^{[87]}$. In ovarian cancer, ATRA has been shown to suppress ALDH1 expression resulting in the attenuation of CSC-like properties in $\mathrm{ALDH}^{+}$cells in vitro and in vivo ${ }^{[8,89]}$. These results suggest that ATRA could be considered as a differentiation therapy for ovarian CSC. Several agents such as metformin and Wnt and ALDH1A inhibitors have been evaluated as potential CSC-targeted therapies. Metformin, a drug that improves glucose tolerance in type II diabetes mellitus, exhibits a favorable effect in various malignancies including ovarian cancer by attenuating tumor growth, potentially due to its suppressive effect on $\mathrm{CSCs}^{[90,91]}$. Low doses of metformin have been shown to selectively inhibit CD $44^{+}$and $\mathrm{CD} 117^{+}$ CSCs in SKOV3 and A2780 ovarian cancer cell lines ${ }^{[92]}$. Furthermore, metformin treatment suppressed $\mathrm{ALDH}^{+}$ovarian CSCs and was additively effective when combined with cisplatin ${ }^{[62]}$. In a phase II trial of 38 ovarian cancer patients with peritoneal dissemination, the median overall survival was 57.9 months after administration of the anticancer drug plus metformin before and after surgery. Tumor burden was reduced 2.4-fold, and increased sensitivity to cisplatin ex vivo was demonstrated in tumors established from $\mathrm{ALDH}^{+} / \mathrm{CD}_{133^{+}} \mathrm{CSC}$ f following metformin treatment ${ }^{[93]}$. Different Wnt pathway inhibitors have been evaluated in ovarian cancer, including salinomycin, a carboxylic acid polyether ionophore antibiotic, which reduces the activity of $\mathrm{ABC}$ transporters, resulting in reduced CSC chemoresistance. Treatment 
with a combination of paclitaxel and salinomycin exhibited growth inhibition of $\mathrm{CD} 44^{+} / \mathrm{CD} 117^{+}$cells in three dimensional-cultured OVCAR3 cells ${ }^{[94]}$, suggesting this combination to be a promising regimen to be evaluated in clinical trials. Given that ALDH activity is a functional marker of ovarian CSCs, several groups have attempted to develop ALDH inhibitors. A recent report revealed that novel pan-ALDH1A family selective inhibitors preferentially target $\mathrm{CD} 133^{+}$ovarian CSCs. These inhibitors upregulate the expression of mitochondrial uncoupling protein (UCP) 1 and 3 and reduce oxidative phosphorylation capacity, resulting in cell programmed necrosis (necroptosis) ${ }^{[95]}$. Given that chemoresistant cells overexpress anti-apoptotic proteins, triggering alternative cell death pathways, such as necroptosis, can lead to better therapeutic outcomes ${ }^{[96]}$. The combination of cisplatin or carboplatin with an ALDH1A inhibitor caused a significant tumor shrinkage in subcutaneous, intraperitoneal, and PDX models of ovarian cancer ${ }^{[95]}$. Treatment with CM37, a small molecule with inhibitory activity against ALDH1 A1, suppressed spheroid proliferation of ovarian cancer cells and reduced expression of OCT4 and SOX2 in $\mathrm{ALDH}^{+}$cells in ascites and ovarian cancer cell lines ${ }^{[97]}$. Importantly, downregulation of only one isozyme from ALDH1A subfamily has been shown to upregulate others, potentially as part of compensatory mechanism, suggesting that pan-ALDH1A inhibition is required to decrease cancer stemness and self-renewal ${ }^{[95]}$. In addition, disulfiram, an ALDH1/2 inhibitor, exerts its anticancer effect by inhibiting ALDH enzymatic activity. This drug promotes the accumulation of reactive oxygen species (ROS) and oxidative stress, subsequently reducing expression of ovarian $\mathrm{ALDH}^{+} / \mathrm{CD} 133^{+} \mathrm{CSC}$ and enhancing cisplatin-induced apoptosis in vitro and in vivo ${ }^{[98,99]}$. Daidzin, an ALDH2 inhibitor, demonstrated no significant toxicity in ovarian cancer cells and did not affect the number of $\mathrm{CD}_{133^{+}}$cells in vitro. Moreover, this drug showed no therapeutic effect in combination with cisplatin in vivo ${ }^{[95]}$, suggesting that $\mathrm{ALDH} 2$ is not associated with cancer stemness and chemoresistance.

\section{CLINICAL STUDIES}

To summarize the novel targeted therapies that are currently being evaluated in clinical studies, a search of the clinicaltrials.gov database was conducted for active trials recruiting as of June 2020. The keywords "resistant" or "targeted therapy" in combination with "recurrent ovarian cancer" were used. A manual search to identify targeted therapies-based clinical trials was performed as well. The types of identified targeted therapies in clinical studies can be broadly classified into four types: (1) immune checkpoint inhibitors; (2) angiogenesis inhibitors; (3) PARPi; and (4) other tyrosine kinase inhibitors [Table 2]. Immune checkpoint inhibitors include program cell death protein 1 (PD-1), program cell death-ligand 1 (PD-L1), program cell death-ligand 2 (PD-L2), and cytotoxic T-lymphocyte-associated protein 4 (CTLA-4) inhibitors. PD-L1 and PD-L2 are highly expressed on CSCs of various cancers ${ }^{[100]}$. The use of anti-PD-L1 immune checkpoint inhibitors targets these ligands and prevents the escape of CSCs from cell death ${ }^{[101]}$. The category of angiogenesis inhibitors that are currently in clinical studies include bevacizumab, sevacizumab, apatinib, cediranib, pazopanib, tivozanib, and VB-111. Anti-angiogenic therapy can induce hypoxia-inducible factor (HIF-1A), which subsequently causes upregulated VEGF production and increased CSCs ${ }^{[102]}$. Therefore, a combination of anti-angiogenic drugs and CSC-targeted therapies may provide promising treatment options ${ }^{[103]}$. The FDA-approved PARPi that are currently evaluated in clinical trials include olaparib, niraparib, and rucaparib. Furthermore, clinical trials of talazoparib, a novel PARPi approved for use in breast cancer, are being conducted for recurrent ovarian cancer patients as well. Treatments with PARPi in ovarian cancer cells in vitro and in vivo resulted in enrichment of $\mathrm{CD} 133^{+}$and $\mathrm{CD} 117^{+} \mathrm{CSC}$, suggesting a need for a combination regimen with CSC-targeted therapies ${ }^{[71]}$. A group of tyrosine kinase inhibitors is comprised of checkpoint kinase 1 (CHK1), ataxia-telangiectasia and Rad3 related protein (ATR), and WEE1 G2 checkpoint kinase (WEE1) inhibitors, which play a pivotal role in DNA damage repair response. These drugs have exhibited an inhibitory effect on CSCs in various cancers in vitro and in vivo, but their effect on ovarian CSCs has not been evaluated ${ }^{[104]}$. In addition, ongoing clinical trials evaluating the effects of different agents include PI3-K inhibitors, AXL receptor tyrosine kinase (AXL) inhibitors, microtubule stabilizers, mitogen-activated protein kinase (MEK) inhibitors, TLR8 agonists, and benzamide histone deacetylase inhibitors. PI3-K inhibitors used in cisplatin-resistant ovarian cancer cell 
Table 2. Clinical trials for platinum-resistant recurrent ovarian cancer

\begin{tabular}{|c|c|c|c|}
\hline & Identifier & Function & Phase \\
\hline \multicolumn{4}{|c|}{ Immune checkpoint inhibitor } \\
\hline \multirow[t]{7}{*}{ Durvalumab } & NCT03026062 & Programmed cell death ligand 1 inhibitor & 2 \\
\hline & NCT03699449 & & 2 \\
\hline & NCT02431559 & & $1 / 2$ \\
\hline & NCT02764333 & & 2 \\
\hline & NCT04019288 & & $1 / 2$ \\
\hline & NCT02963831 & & 2 \\
\hline & NCT02811497 & & 2 \\
\hline Pembrolizumab & NCT02608684 & Targets programmed cell death protein 1 receptor & 2 \\
\hline Atezolizumab & NCT03363867 & Targets programmed cell death ligand 1 (PD-L1) & 2 \\
\hline TSR-042 & NCT03574779 & Anti-PD1 antibody & 2 \\
\hline \multirow[t]{2}{*}{ Tremelimumab } & NCT02953457 & Activates the immune system by targeting CTLA-4 & 2 \\
\hline & NCT03026062 & & 2 \\
\hline Ipilimumab & NCT03508570 & Activates the immune system by targeting CTLA-4 & 1 \\
\hline Avelumab & NCT02580058 & Targets the protein programmed death-ligand 1 (PD-L1) & 3 \\
\hline \multicolumn{4}{|c|}{ Anti-angiogenic inhibitor } \\
\hline Apatinib & NCT04348032 & Vascular endothelial growth factor receptor-2 (VEGFR2) inhibitor & 2 \\
\hline Cediranib & NCT03699449 & Vascular endothelial growth factor inhibitor & 2 \\
\hline Anlotinib & NCT04376073 & c-MET/TIE-2/VEGFR inhibitor & 2 \\
\hline Tivozanib & NCT01853644 & Oral VEGF receptor tyrosine kinase inhibitor & 2 \\
\hline Bevacizumab & NCT03093155 & VEGF receptor tyrosine kinase inhibitor & 2 \\
\hline Sevacizumab & NCT03763123 & VEGF receptor tyrosine kinase inhibitor & 1 \\
\hline VB-111 & NCT03398655 & An anti-angiogenic gene therapy & 3 \\
\hline Regorafenib & NCT02736305 & Dual-targeted VEGFR2-TIE2 tyrosine kinase inhibition & 2 \\
\hline Pazopanib & NCT01402271 & $\begin{array}{l}\text { Selective multi-targeted receptor tyrosine kinase inhibitor that } \\
\text { blocks tumor growth and inhibits angiogenesis }\end{array}$ & $1 / 2$ \\
\hline \multicolumn{4}{|l|}{ PARP inhibitor } \\
\hline \multirow[t]{8}{*}{ Olaparib } & NCT02889900 & PARP inhibitor & 2 \\
\hline & NCT04633239 & & 1 \\
\hline & NCT03117933 & & 2 \\
\hline & NCT02898207 & & 1 \\
\hline & NCT03161132 & & 2 \\
\hline & NCT03699449 & & 2 \\
\hline & NCT03314740 & & 2 \\
\hline & NCT02502266 & & $2 / 3$ \\
\hline \multirow[t]{7}{*}{ Niraparib } & NCT04376073 & PARP inhibitor & 2 \\
\hline & NCT03955471 & & 2 \\
\hline & NCT04502602 & & 1 \\
\hline & NCT03586661 & & 1 \\
\hline & NCT04217798 & & 2 \\
\hline & NCT03944902 & & 1 \\
\hline & NCT01227941 & & 1 \\
\hline Rucaparib & NCT03552471 & PARP inhibitor & 1 \\
\hline Talazoparib & NCT03330405 & PARP inhibitor & 2 \\
\hline \multicolumn{4}{|c|}{ Other tyrosine kinase inhibitor } \\
\hline BAY1895344 & NCT04267939 & Ataxia-telangiectasia and Rad3 related protein (ATR) inhibitor & 1 \\
\hline Prexasertib & NCT03414047 & CHK1 inhibitor & 2 \\
\hline Adavosertib & NCT03579316 & WEE1 G2 checkpoint kinase (WEE1) inhibitor & 2 \\
\hline Copanlisib & NCT03586661 & Phosphatidylinositol-3-kinase (PI3K) inhibitor & 1 \\
\hline TP-0903 & NCT02729298 & $\begin{array}{l}\text { Targets the AXL (derived from the Greek word "anexelekto", } \\
\text { meaning uncontrolled) receptor tyrosine kinase }\end{array}$ & 1 \\
\hline AVB-S6-500 & NCT03639246 & $\begin{array}{l}\text { Targets the AXL (derived from the Greek word "anexelekto", } \\
\text { meaning uncontrolled) receptor tyrosine kinase }\end{array}$ & $1 / 2$ \\
\hline \multirow[t]{2}{*}{ Ixabepilone } & NCT03093155 & Stabilizes microtubules & 2 \\
\hline & NCT02595892 & & 2 \\
\hline \multirow[t]{2}{*}{ Cobimetinib } & NCT03363867 & MEK inhibitor & 2 \\
\hline & NCT02101775 & & 2 \\
\hline Motolimod & NCT02431559 & Toll-like receptor 8 (TLR8) agonist & $1 / 2$ \\
\hline Entinostat & NCT03924245 & Benzamide histone deacetylase inhibitor & $1 / 2$ \\
\hline
\end{tabular}


lines exhibited reduction in CD44variant6, CD117, ALDH1A1, and Snail expression ${ }^{[105]}$. Interestingly, in breast cancer, inhibition of AXL increased CSC chemosensitivity ${ }^{[106]}$, whereas histone deacetylase inhibitors manifested preferential targeting of breast CSCs, suggesting a potential therapeutic effect in ovarian CSCs as well ${ }^{[107]}$. In addition, a recent study of high-grade serous ovarian cancer revealed that an MEK1/2 inhibitor, trametinib, arrests cell proliferation but also enriches cancer stemness, suggesting a need for a combination regimen with CSC-targeted therapy ${ }^{[108]}$.

\section{CONCLUSION}

Ovarian CSCs are a population of cells that are often enriched in residual tumors following initial treatments with conventional chemotherapy. These CSCs are directly associated with acquired chemoresistance in ovarian cancer. Various studies have attempted to assess CSCs as prognostic markers using various laboratory techniques such as qPCR, immunochemistry, and FACS, to quantify mRNA and protein expression levels or enzymatic activity. This approach (using different techniques for evaluation) has introduced a lot of variations as mRNA level does not necessarily correlate with protein level or enzymatic activity. In addition, the ALDH1 A antibody extensively used for immunochemical analysis does not differentiate between ALDH1 A1, 1A2, and $1 \mathrm{~A} 3$ isozymes. Thus, a more rigorous experimental design is needed to evaluate single markers and dual CSC marker combinations to insure reproducible results. A FACS-based assay for ALDH activity is affected by cell confluence and cell number used in each assay, raising the importance of standardized protocols to ensure consistent measurements amongst research groups.

Given the CSC importance in prognosis and disease progression, the molecular biology of ovarian CSCs needs to be further elucidated to design novel CSC-targeted therapies. Targeted therapies against CSCs have the potential to reduce tumor growth and improve patient prognosis. On the basis of recent findings, the combination of chemotherapy and CSC-targeted therapy may be one of the most promising anticancer treatments for ovarian cancer. Currently, many clinical trials are ongoing to evaluate conventional chemotherapy in combination with various targeted therapies in platinum-resistant ovarian tumors, which potentially will change the treatment strategy for patients with recurrent ovarian cancer. Finally, assessing how novel targeted therapies affect the various CSC populations is required to rationally design new treatment regimens.

\section{DECLARATIONS}

\section{Authors' contributions}

Performed the literature search and wrote the first draft: Takahashi A

Advised on clinical matters and edited a last draft: Hong L

Conceived the article, edited all drafts, received funding, and reviewed the final draft: Chefetz I

\section{Availability of data and materials}

Not applicable.

\section{Financial support and sponsorship}

The work was supported by the Liz Tilberis early career OCRA award, the Marsha Rivkin Ovarian Center pilot award, the WeRoc research grant from Foundation for Women's Cancer, and the Elsa U. Pardee Foundation (awards to Chefetz I).

\section{Conflicts of interest}

All authors declared that there are no conflicts of interest. 


\section{Ethical approval and consent to participate}

Not applicable.

\section{Consent for publication}

Not applicable.

\section{Copyright}

(c) The Author(s) 2020.

\section{REFERENCES}

1. Colombo N, Sessa C, du Bois A, et al. ESMO-ESGO consensus conference recommendations on ovarian cancer: pathology and molecular biology, early and advanced stages, borderline tumours and recurrent disease $\uparrow$. Ann Oncol 2019;30:672-705.

2. Torre LA, Trabert B, DeSantis CE, Miller KD, Samimi G, et al. Ovarian cancer statistics, 2018. CA Cancer J Clin 2018;68:284-96.

3. Kaku T, Ogawa S, Kawano Y, et al. Histological classification of ovarian cancer. Med Electron Microsc 2003;36:9-17.

4. Shih IM, Kurman RJ. Ovarian tumorigenesis: a proposed model based on morphological and molecular genetic analysis. Am J Pathol 2004;164:1511-8.

5. Alsop K, Fereday S, Meldrum C, et al. BRCA mutation frequency and patterns of treatment response in BRCA mutation-positive women with ovarian cancer: a report from the Australian Ovarian Cancer Study Group. J Clin Oncol 2012;30:2654-63.

6. Jayson GC, Kohn EC, Kitchener HC, Ledermann JA. Ovarian cancer. Lancet 2014;384:1376-88.

7. Seidman JD, Yemelyanova A, Cosin JA, Smith A, Kurman RJ. Survival rates for international federation of gynecology and obstetrics stage III ovarian carcinoma by cell type: a study of 262 unselected patients with uniform pathologic review. Int J Gynecol Cancer 2012;22:367-71.

8. du Bois A, Reuss A, Pujade-Lauraine E, Harter P, Ray-Coquard I, Pfisterer J. Role of surgical outcome as prognostic factor in advanced epithelial ovarian cancer: a combined exploratory analysis of 3 prospectively randomized phase 3 multicenter trials. Cancer 2009;115:1234-44.

9. Marth C, Reimer D, Zeimet AG. Front-line therapy of advanced epithelial ovarian cancer: standard treatment. Ann Oncol 2017;28 Suppl 8:viii36-9.

10. Muggia F. Platinum compounds 30 years after the introduction of cisplatin: implications for the treatment of ovarian cancer. Gynecol Oncol 2009;112:275-81.

11. Rubin SC, Randall TC, Armstrong KA, Chi DS, Hoskins WJ. Ten-year follow-up of ovarian cancer patients after second-look laparotomy with negative findings. Obstet Gynecol 1999;93:21-4.

12. Ushijima K. Treatment for recurrent ovarian cancer-at first relapse. J Oncol 2010;2010:497429.

13. Takahashi A, Kato K, Matsuura M, et al. Comparison of secondary cytoreductive surgery plus chemotherapy with chemotherapy alone for recurrent epithelial ovarian, tubal, or peritoneal carcinoma: a propensity score-matched analysis of 112 consecutive patients. Medicine (Baltimore) 2017;96:e8006.

14. Burger RA, Brady MF, Bookman MA, et al; Gynecologic Oncology Group. Incorporation of bevacizumab in the primary treatment of ovarian cancer. $N$ Engl J Med 2011;365:2473-83.

15. Moore K, Colombo N, Scambia G, et al. Maintenance olaparib in patients with newly diagnosed advanced ovarian cancer. $N$ Engl $J$ Med 2018;379:2495-505.

16. Perren TJ, Swart AM, Pfisterer J, et al; ICON7 Investigators. A phase 3 trial of bevacizumab in ovarian cancer. $N$ Engl $J$ Med 2011;365:2484-96

17. Jiang X, Li X, Li W, Bai H, Zhang Z. PARP inhibitors in ovarian cancer: sensitivity prediction and resistance mechanisms. $J$ Cell Mol Med 2019;23:2303-13.

18. Kim A, Ueda Y, Naka T, Enomoto T. Therapeutic strategies in epithelial ovarian cancer. J Exp Clin Cancer Res 2012;31:14.

19. Au KK, Josahkian JA, Francis JA, Squire JA, Koti M. Current state of biomarkers in ovarian cancer prognosis. Future Oncol 2015;11:3187-95.

20. Hanker LC, Loibl S, Burchardi N, et al; AGO and GINECO study group. The impact of second to sixth line therapy on survival of relapsed ovarian cancer after primary taxane/platinum-based therapy. Ann Oncol 2012;23:2605-12.

21. Chen W, Dong J, Haiech J, Kilhoffer MC, Zeniou M. Cancer stem cell quiescence and plasticity as major challenges in cancer therapy. Stem Cells Int 2016;2016:1740936.

22. Takeishi S, Nakayama KI. To wake up cancer stem cells, or to let them sleep, that is the question. Cancer Sci 2016;107:875-81.

23. Gao Y, Foster R, Yang X, et al. Up-regulation of CD44 in the development of metastasis, recurrence and drug resistance of ovarian cancer. Oncotarget 2015;6:9313-26.

24. Lapidot T, Sirard C, Vormoor J, et al. A cell initiating human acute myeloid leukaemia after transplantation into SCID mice. Nature 1994;367:645-8.

25. Al-Hajj M, Wicha MS, Benito-Hernandez A, Morrison SJ, Clarke MF. Prospective identification of tumorigenic breast cancer cells. Proc Natl Acad Sci U S A 2003;100:3983-8. 
26. Singh SK, Clarke ID, Terasaki M, et al. Identification of a cancer stem cell in human brain tumors. Cancer Res 2003;63:5821-8.

27. Bapat SA, Mali AM, Koppikar CB, Kurrey NK. Stem and progenitor-like cells contribute to the aggressive behavior of human epithelial ovarian cancer. Cancer Res 2005;65:3025-9.

28. Zhang S, Balch $\mathrm{C}$, Chan MW, et al. Identification and characterization of ovarian cancer-initiating cells from primary human tumors. Cancer Res 2008;68:4311-20.

29. Curley MD, Therrien VA, Cummings CL, et al. CD133 expression defines a tumor initiating cell population in primary human ovarian cancer. Stem Cells 2009;27:2875-83.

30. Alvero AB, Fu HH, Holmberg J, et al. Stem-like ovarian cancer cells can serve as tumor vascular progenitors. Stem Cells 2009;27:2405-13.

31. Wei X, Dombkowski D, Meirelles K, et al. Mullerian inhibiting substance preferentially inhibits stem/progenitors in human ovarian cancer cell lines compared with chemotherapeutics. Proc Natl Acad Sci U S A 2010;107:18874-9.

32. Kryczek I, Liu S, Roh M, et al. Expression of aldehyde dehydrogenase and CD133 defines ovarian cancer stem cells. Int J Cancer 2012;130:29-39.

33. Meng E, Long B, Sullivan P, et al. CD44+/CD24- ovarian cancer cells demonstrate cancer stem cell properties and correlate to survival. Clin Exp Metastasis 2012;29:939-48.

34. Cao L, Shao M, Schilder J, Guise T, Mohammad KS, Matei D. Tissue transglutaminase links TGF- $\beta$, epithelial to mesenchymal transition and a stem cell phenotype in ovarian cancer. Oncogene 2012;31:2521-34.

35. Shah V, Taratula O, Garbuzenko OB, Taratula OR, Rodriguez-Rodriguez L, Minko T. Targeted nanomedicine for suppression of CD44 and simultaneous cell death induction in ovarian cancer: an optimal delivery of siRNA and anticancer drug. Clin Cancer Res 2013;19:6193-204.

36. Akhter MZ, Sharawat SK, Kumar V, et al. Aggressive serous epithelial ovarian cancer is potentially propagated by EpCAM. Oncogene 2018;37:2089-103.

37. Chefetz I, Alvero AB, Holmberg JC, et al. TLR2 enhances ovarian cancer stem cell self-renewal and promotes tumor repair and recurrence. Cell Cycle 2013;12:511-21.

38. Ruan Z, Liu J, Kuang Y. Isolation and characterization of side population cells from the human ovarian cancer cell line SK-OV-3. Exp Ther Med 2015;10:2071-8.

39. Boesch M, Zeimet AG, Reimer D, et al. The side population of ovarian cancer cells defines a heterogeneous compartment exhibiting stem cell characteristics. Oncotarget 2014;5:7027-39.

40. Alvero AB, Chen R, Fu HH, et al. Molecular phenotyping of human ovarian cancer stem cells unravels the mechanisms for repair and chemoresistance. Cell Cycle 2009;8:158-66.

41. Tsuneki M, Madri JA. CD44 regulation of endothelial cell proliferation and apoptosis via modulation of CD31 and VE-cadherin expression. J Biol Chem 2014;289:5357-70.

42. Silasi DA, Alvero AB, Illuzzi J, et al. MyD88 predicts chemoresistance to paclitaxel in epithelial ovarian cancer. Yale J Biol Med 2006;79:153-63.

43. Ponta H, Sherman L, Herrlich PA. CD44: from adhesion molecules to signalling regulators. Nat Rev Mol Cell Biol 2003;4:33-45.

44. Cioffi M, D'Alterio C, Camerlingo R, et al. Identification of a distinct population of CD133(+)CXCR4(+) cancer stem cells in ovarian cancer. Sci Rep 2015;5:10357.

45. Kusumbe AP, Mali AM, Bapat SA. CD133-expressing stem cells associated with ovarian metastases establish an endothelial hierarchy and contribute to tumor vasculature. Stem Cells 2009;27:498-508.

46. Sharrow AC, Perkins B, Collector MI, Yu W, Simons BW, Jones RJ. Characterization of aldehyde dehydrogenase 1 high ovarian cancer cells: Towards targeted stem cell therapy. Gynecol Oncol 2016;142:341-8.

47. Silva IA, Bai S, McLean K, et al. Aldehyde dehydrogenase in combination with CD133 defines angiogenic ovarian cancer stem cells that portend poor patient survival. Cancer Res 2011;71:3991-4001.

48. Liao J, Qian F, Tchabo N, et al. Ovarian cancer spheroid cells with stem cell-like properties contribute to tumor generation, metastasis and chemotherapy resistance through hypoxia-resistant metabolism. PLoS One 2014;9:e84941.

49. Kuroda T, Hirohashi Y, Torigoe T, et al. ALDH1-high ovarian cancer stem-like cells can be isolated from serous and clear cell adenocarcinoma cells, and ALDH1 high expression is associated with poor prognosis. PLoS One 2013;8:e65158.

50. Ishiguro T, Sato A, Ohata H, et al. Establishment and characterization of an in vitro model of ovarian cancer stem-like cells with an enhanced proliferative capacity. Cancer Res 2016;76:150-60.

51. Gao MQ, Choi YP, Kang S, Youn JH, Cho NH. CD24+ cells from hierarchically organized ovarian cancer are enriched in cancer stem cells. Oncogene 2010;29:2672-80.

52. Luo L, Zeng J, Liang B, et al. Ovarian cancer cells with the CD117 phenotype are highly tumorigenic and are related to chemotherapy outcome. Exp Mol Pathol 2011;91:596-602.

53. Wen Y, Hou Y, Huang Z, Cai J, Wang Z. SOX2 is required to maintain cancer stem cells in ovarian cancer. Cancer Sci 2017;108:719-31.

54. Belotte J, Fletcher NM, Alexis M, et al. Sox2 gene amplification significantly impacts overall survival in serous epithelial ovarian cancer. Reprod Sci 2015;22:38-46.

55. Curley MD, Garrett LA, Schorge JO, Foster R, Rueda BR. Evidence for cancer stem cells contributing to the pathogenesis of ovarian cancer. Front Biosci (Landmark Ed) 2011;16:368-92.

56. Burgos-Ojeda D, Rueda BR, Buckanovich RJ. Ovarian cancer stem cell markers: prognostic and therapeutic implications. Cancer Lett 2012;322:1-7.

57. Tao Y, Li H, Huang R, et al. Clinicopathological and Prognostic Significance of Cancer Stem Cell Markers in Ovarian Cancer Patients: 
Evidence from 52 Studies. Cell Physiol Biochem 2018;46:1716-26.

58. Tayama S, Motohara T, Narantuya D, et al. The impact of EpCAM expression on response to chemotherapy and clinical outcomes in patients with epithelial ovarian cancer. Oncotarget 2017;8:44312-25.

59. Di J, Duiveman-de Boer T, Zusterzeel PL, Figdor CG, Massuger LF, Torensma R. The stem cell markers Oct4A, Nanog and c-Myc are expressed in ascites cells and tumor tissue of ovarian cancer patients. Cell Oncol (Dordr) 2013;36:363-74.

60. Peng S, Maihle NJ, Huang Y. Pluripotency factors Lin28 and Oct4 identify a sub-population of stem cell-like cells in ovarian cancer. Oncogene 2010;29:2153-9.

61. Baker VV, Borst MP, Dixon D, Hatch KD, Shingleton HM, Miller D. c-myc amplification in ovarian cancer. Gynecol Oncol1990;38:340-2.

62. Vita M, Henriksson M. The Myc oncoprotein as a therapeutic target for human cancer. Semin Cancer Biol 2006;16:318-30.

63. Cancer Genome Atlas Research Network. Integrated genomic analyses of ovarian carcinoma. Nature 2011;474:609-15.

64. Reyes-González JM, Armaiz-Peña GN, Mangala LS, et al. Targeting c-MYC in platinum-resistant ovarian cancer. Mol Cancer Ther 2015;14:2260-9.

65. Wang Y, Cardenas H, Fang F, et al. Epigenetic targeting of ovarian cancer stem cells. Cancer Res 2014;74:4922-36.

66. Latifi A, Luwor RB, Bilandzic M, et al. Isolation and characterization of tumor cells from the ascites of ovarian cancer patients: molecular phenotype of chemoresistant ovarian tumors. PLoS One 2012;7:e46858.

67. Steg AD, Bevis KS, Katre AA, et al. Stem cell pathways contribute to clinical chemoresistance in ovarian cancer. Clin Cancer Res 2012;18:869-81.

68. Bellio C, DiGloria C, Spriggs DR, Foster R, Growdon WB, Rueda BR. The metabolic inhibitor CPI-613 negates treatment enrichment of ovarian cancer stem cells. Cancers (Basel) 2019;11:1678.

69. Abubaker K, Latifi A, Luwor R, et al. Short-term single treatment of chemotherapy results in the enrichment of ovarian cancer stem celllike cells leading to an increased tumor burden. Mol Cancer 2013;12:24.

70. Zhou N, Wu X, Yang B, Yang X, Zhang D, Qing G. Stem cell characteristics of dormant cells and cisplatin-induced effects on the stemness of epithelial ovarian cancer cells. Mol Med Rep 2014;10:2495-504.

71. Bellio C, DiGloria C, Foster R, et al. PARP Inhibition induces enrichment of DNA repair-proficient CD133 and CD117 positive ovarian cancer stem cells. Mol Cancer Res 2019;17:431-45.

72. Chau WK, Ip CK, Mak AS, Lai HC, Wong AS. c-Kit mediates chemoresistance and tumor-initiating capacity of ovarian cancer cells through activation of Wnt/ $\beta$-catenin-ATP-binding cassette G2 signaling. Oncogene 2013;32:2767-81.

73. Fischer MM, Cancilla B, Yeung VP, et al. WNT antagonists exhibit unique combinatorial antitumor activity with taxanes by potentiating mitotic cell death. Sci Adv 2017;3:e1700090.

74. Song X, Yan L, Lu C, et al. Activation of hedgehog signaling and its association with cisplatin resistance in ovarian epithelial tumors. Oncol Lett 2018;15:5569-76.

75. Park JT, Chen X, Tropè CG, Davidson B, Shih IeM, Wang TL. Notch3 overexpression is related to the recurrence of ovarian cancer and confers resistance to carboplatin. Am J Pathol 2010;177:1087-94.

76. Jung SG, Kwon YD, Song JA, et al. Prognostic significance of Notch 3 gene expression in ovarian serous carcinoma. Cancer Sci 2010;101:1977-83.

77. Martins FC, Santiago Id, Trinh A, et al. Combined image and genomic analysis of high-grade serous ovarian cancer reveals PTEN loss as a common driver event and prognostic classifier. Genome Biol 2014;15:526.

78. Russo A, Czarnecki AA, Dean M, et al. PTEN loss in the fallopian tube induces hyperplasia and ovarian tumor formation. Oncogene 2018;37:1976-90.

79. Honig A, Hahne JC, Meyer S, et al. PI3K inhibitor D-116883 is effective in in vitro models of ovarian cancer. Anticancer Res 2012;32:2035-41.

80. Westfall SD, Skinner MK. Inhibition of phosphatidylinositol 3-kinase sensitizes ovarian cancer cells to carboplatin and allows adjunct chemotherapy treatment. Mol Cancer Ther 2005;4:1764-71.

81. Carden CP, Stewart A, Thavasu P, et al. The association of PI3 kinase signaling and chemoresistance in advanced ovarian cancer. Mol Cancer Ther 2012;11:1609-17.

82. Dobbin ZC, Landen CN. The importance of the PI3K/AKT/MTOR pathway in the progression of ovarian cancer. Int $J$ Mol Sci 2013;14:8213-27.

83. Chen R, Alvero AB, Silasi DA, et al. Regulation of IKKbeta by miR-199a affects NF-kappaB activity in ovarian cancer cells. Oncogene 2008;27:4712-23.

84. Zou Z, Yuan Z, Zhang Q, et al. Aurora kinase A inhibition-induced autophagy triggers drug resistance in breast cancer cells. Autophagy 2012;8:1798-810

85. Chefetz I, Holmberg JC, Alvero AB, Visintin I, Mor G. Inhibition of Aurora-A kinase induces cell cycle arrest in epithelial ovarian cancer stem cells by affecting NFKB pathway. Cell Cycle 2011;10:2206-14.

86. Tallman MS, Andersen JW, Schiffer CA, et al. All-trans-retinoic acid in acute promyelocytic leukemia. N Engl J Med 1997;337:1021-8.

87. Sporn MB, Roberts AB. Role of retinoids in differentiation and carcinogenesis. Cancer Res 1983;43:3034-40.

88. Kim D, Choi BH, Ryoo IG, Kwak MK. High NRF2 level mediates cancer stem cell-like properties of aldehyde dehydrogenase (ALDH)high ovarian cancer cells: inhibitory role of all-trans retinoic acid in ALDH/NRF2 signaling. Cell Death Dis 2018;9:896.

89. Whitworth JM, Londoño-Joshi AI, Sellers JC, et al. The impact of novel retinoids in combination with platinum chemotherapy on ovarian cancer stem cells. Gynecol Oncol 2012;125:226-30. 
90. Shank JJ, Yang K, Ghannam J, et al. Metformin targets ovarian cancer stem cells in vitro and in vivo. Gynecol Oncol 2012;127:390-7.

91. Del Barco S, Vazquez-Martin A, Cufí S, et al. Metformin: multi-faceted protection against cancer. Oncotarget 2011;2:896-917.

92. Zhang R, Zhang P, Wang H, et al. Inhibitory effects of metformin at low concentration on epithelial-mesenchymal transition of CD44(+) CD117(+) ovarian cancer stem cells. Stem Cell Res Ther 2015;6:262.

93. Brown JR, Chan DK, Shank JJ, et al. Phase II clinical trial of metformin as a cancer stem cell-targeting agent in ovarian cancer. JCI Insight 2020;5:133247.

94. Chung H, Kim YH, Kwon M, et al. The effect of salinomycin on ovarian cancer stem-like cells. Obstet Gynecol Sci 2016;59:261-8.

95. Chefetz I, Grimley E, Yang K, et al. A Pan-ALDH1A inhibitor induces necroptosis in ovarian cancer stem-like cells. Cell Rep 2019;26:3061-3075.e6.

96. Khan I, Yousif A, Chesnokov M, Hong L, Chefetz I. A decade of cell death studies: Breathing new life into necroptosis. Pharmacol Ther 2020;107717.

97. Nwani NG, Condello S, Wang Y, et al. A novel ALDH1A1 inhibitor targets cells with stem cell characteristics in ovarian cancer. Cancers (Basel) 2019;11:502.

98. Harrington BS, Ozaki MK, Caminear MW, et al. Drugs targeting tumor-initiating cells prolong survival in a post-surgery, postchemotherapy ovarian cancer relapse model. Cancers (Basel) 2020;12:1645.

99. Guo F, Yang Z, Kulbe H, Albers AE, Sehouli J, Kaufmann AM. Inhibitory effect on ovarian cancer ALDH+ stem-like cells by Disulfiram and Copper treatment through ALDH and ROS modulation. Biomed Pharmacother 2019;118:109371.

100. Wu Y, Chen M, Wu P, Chen C, Xu ZP, Gu W. Increased PD-L1 expression in breast and colon cancer stem cells. Clin Exp Pharmacol Physiol 2017;44:602-4.

101. Codd AS, Kanaseki T, Torigo T, Tabi Z. Cancer stem cells as targets for immunotherapy. Immunology 2018;153:304-14.

102. Heddleston JM, Li Z, Lathia JD, Bao S, Hjelmeland AB, Rich JN. Hypoxia inducible factors in cancer stem cells. Br J Cancer 2010;102:789-95.

103. Markowska A, Sajdak S, Markowska J, Huczyński A. Angiogenesis and cancer stem cells: New perspectives on therapy of ovarian cancer. Eur J Med Chem 2017;142:87-94.

104. Ronco C, Martin AR, Demange L, Benhida R. ATM, ATR, CHK1, CHK2 and WEE1 inhibitors in cancer and cancer stem cells. Medchemcomm 2017;8:295-319.

105. Deng J, Bai X, Feng X, et al. Inhibition of PI3K/Akt/mTOR signaling pathway alleviates ovarian cancer chemoresistance through reversing epithelial-mesenchymal transition and decreasing cancer stem cell marker expression. BMC Cancer 2019;19:618.

106. Asiedu MK, Beauchamp-Perez FD, Ingle JN, Behrens MD, Radisky DC, Knutson KL. AXL induces epithelial-to-mesenchymal transition and regulates the function of breast cancer stem cells. Oncogene 2014;33:1316-24.

107. Witt AE, Lee CW, Lee TI, et al. Identification of a cancer stem cell-specific function for the histone deacetylases, HDAC1 and HDAC7, in breast and ovarian cancer. Oncogene 2017;36:1707-20.

108. Chesnokov M, Khan I, Park Y, et al. The MEK1/2 pathway as a therapeutic target in high-grade serous ovarian carcinoma. bioRxiv 2019:772061. 